\title{
Synthesis of New Binary Thiazole-Based Heterocycles and Their Molecular Docking Study as COVID-19 Main Protease (Mpro) Inhibitors
}

\author{
E. Abdel-Latif ${ }^{a, *}$, T. K. Khatab ${ }^{a}$, A. Fekri ${ }^{a}$, and M. E. Khalifa ${ }^{b}$ \\ ${ }^{a}$ Department of Chemistry, Faculty of Science, Mansoura University, Mansoura, 35516 Egypt \\ ${ }^{b}$ Department of Chemistry, College of Science, Taif University, P.O. Box 11099, Taif, 21944 Saudi Arabia \\ *e-mail: ehabattia00@gmx.net
}

Received May 19, 2021; revised June 14, 2021; accepted June 14, 2021

\begin{abstract}
Isolated polynuclear binary heterocyclic compounds containing thiazole building block combined with benzofuran, pyrrole, thiazole, or thiophene via carboxamide and/or secondary amine as a junction are presented. The synthetic strategy of those is based on utilization of 2-chloroacetamido-4-phenylthiazole in the synthesis of binary heterocyclic compounds by cyclocondensation with salicylic aldehyde, acetonitrile derivatives, ammonium thiocyanate, 3-mercaptoacrylonitrile derivatives, and/or 3-mercaptoacrylate derivatives. The prepared binary thiazole-based heterocycles have been studied as protease $\left(\mathrm{M}^{\text {pro }}\right)$ inhibitors by molecular docking for visualization of their orientation and interactions with COVID-19 units using hydroxychloroquine as a reference molecule.
\end{abstract}

Keywords: binary heterocycles, chloroacetamide, thiazole, benzofuran, protease, COVID-19

DOI: $10.1134 / \mathrm{S} 1070363221090231$

\section{INTRODUCTION}

Polynuclear heterocycles containing thiazole ring are considered as a specific scaffolds in the synthesis of pharmacologically active compounds and efficient drugs [1]. Thiazole derivatives are components of molecules that demonstrate a broad spectrum of biologically activities [2-6]. For example, the efficient drug Famotidine is used in treatment of peptic ulcer and controlling gastro esophageal reflux [7], Cefdinir is the third generation broad spectrum cephalosporin antibiotic [8]. The biological and synthetic significance of aminothiazole derivatives stimulated our design of some new molecules for COVID-19 main protease $\left(\mathrm{M}^{\mathrm{pro}}\right)$ inhibitor based on molecular docking studies.

Covid-19 is considered as one of the biggest disasters that occurred in the modern era of mankind [9]. Up to now, no decisive treatment of it has been found yet. In modern drug discovery the molecular docking tools are considered as an important approach to design of agonists of drugs by calculating the ligand-receptor affinity.

\section{RESULTS AND DISCUSSION}

2-Chloroacetamido-4-phenylthiazole (2) has been synthesized in $75 \%$ yield by the earlier developed method [10]. Reactions of the intermediate 2 with various nucleophiles led to new heterocyclic structures containing thiazole nucleus linked with furan, pyrrole, thiazole, and/or thiophene ring system. Reaction of compound $\mathbf{2}$ with salicylaldehyde and anhydrous $\mathrm{K}_{2} \mathrm{CO}_{3}$ in dimethyl sulfoxide led to the corresponding $\mathrm{N}$-(4-phenylthiazol2-yl)benzofuran-2-carboxamide (3) (Scheme 1). The reaction of compound $\mathbf{2}$ with acetonitrile derivatives (malononitrile, cyanoacetamide and 2-benzothiazolyl acetonitrile) in presence of sodium ethoxide gave the corresponding 3-substituted-1-(4-phenylthiazol-2-yl)pyrroles $4 \mathbf{a}-\mathbf{4 c}$. Chemical structures of the products $\mathbf{4 a}-\mathbf{4 c}$ were elucidated from their elemental analyses and spectroscopic data. Refluxing of compound $\mathbf{2}$ with ammonium thiocyanate in ethanol afforded 2-[(4-phenylthiazol-2-yl)imino]thiazolidin-4-one (5), which underwent the reaction with $p$-chlorobenzaldehyde in $\mathrm{AcOH}$ and fused $\mathrm{AcONa}$ to furnish the corresponding condensation product 5-(4-chlorobenzylidene)-2-[(4phenylthiazol-2-yl)imino]thiazolidin-4-one (6). Chemical structures of thiazolidin-4-one derivatives $\mathbf{5}$ and $\mathbf{6}$ were based on elemental analysis and spectral data.

Cyclocondensation of compound 2 with 3-mercapto3-phenylamino-acrylonitrile derivatives $\mathbf{7 a}-\mathbf{7} \mathbf{c}$ under the 
Scheme 1. Reactions of 2-chloroacetamido-4-phenylthiazole (2) with salicylaldehyde, activated nitriles and ammonium thiocyanate.

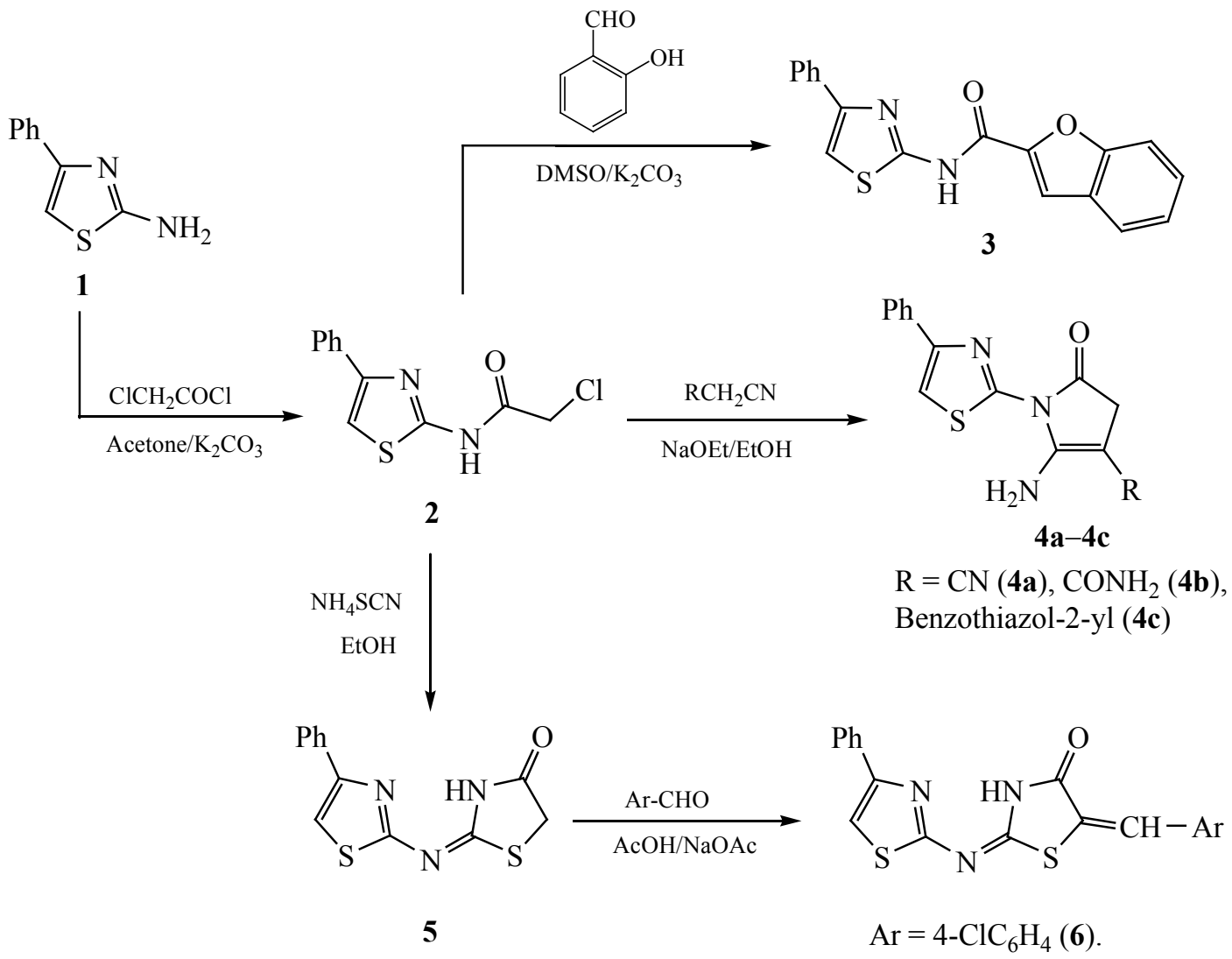

Scheme 2. Synthesis of 3-amino-5-phenylamino-2-(4-phenylthiazol-2-yl-carbamoyl)thiophene derivatives 9a-9c.<smiles>O=C(CCl)Nc1nc(-c2ccccc2)cs1</smiles>

2<smiles>[R]C(C#N)=C(S)Nc1ccccc1</smiles>
7

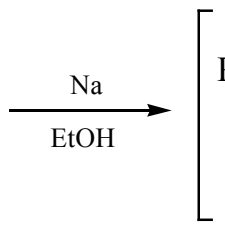

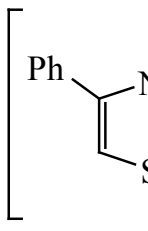<smiles>Cc1csc(N)n1</smiles><smiles>[R]C(C#N)=C(Nc1ccccc1)SCC(N)=O</smiles>

8<smiles>[R]c1c(Nc2ccccc2)sc(C(=O)Nc2nc(-c3ccccc3)cs2)c1N</smiles><smiles>CCC</smiles>

9a-9c

$\mathrm{R}=\mathrm{CN}(\mathbf{9 a}), \mathrm{CONH}_{2}(\mathbf{9 b})$, Benzothiazol-2-yl (9c).

action of sodium ethoxide. The corresponding 3-amino5-phenylamino-2-(4-phenylthiazol-2-yl-carbamoyl)-4substitutedthiophene derivatives $9 \mathbf{9}-9 \mathbf{c}$ were formed. Formation of thiophene derivatives 9 could proceed via nucleophilic attack of the thiol function leading to the sulfide intermediate 8, which upon intramolecular cyclization via nucleophilic addition of the methylene group to the activated nitrile could produce the corresponding 3-amino-4-substituted-thiophenes 9 (Scheme 2). IR, NMR and mass spectra of the products 9a-9c were in agreement with their molecular structures. 
Scheme 3. Synthesis of 3-hydroxy-5-phenylamino-2-(4-phenylthiazol-2-yl-carbamoyl)thiophene derivative 12.

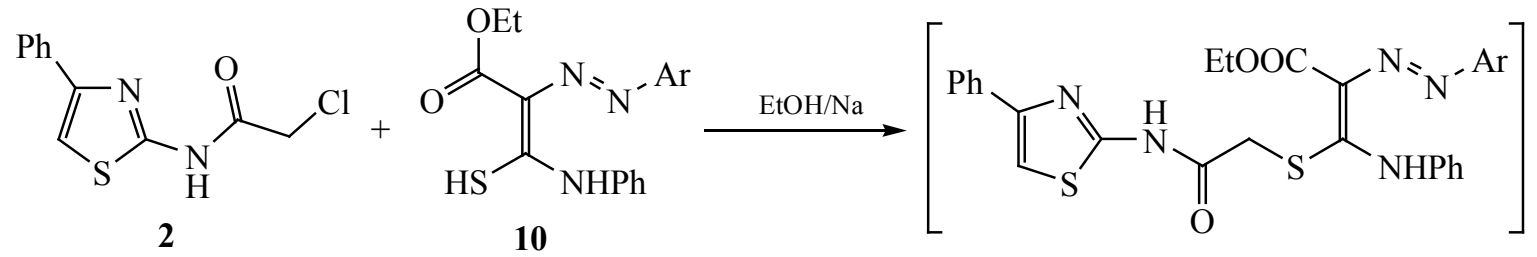

11<smiles>O=C(Nc1nc(-c2ccccc2)cs1)c1sc(Nc2ccccc2)c(N=N[AlH2])c1O</smiles>

12

Scheme 4. Synthesis of 3-methyl-5-phenylamino-2-(4-phenylthiazol-2-yl-carbamoyl)thiophene derivative 15.<smiles>O=C(CCl)Nc1nc(-c2ccccc2)cs1</smiles>

2

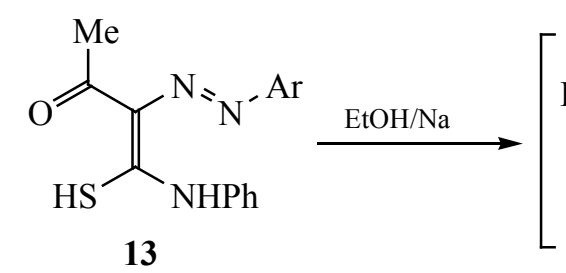

13<smiles>Cc1c(C(=O)Nc2nc(-c3ccccc3)cs2)sc(Nc2ccccc2)c1N=N[Al]</smiles>

Refluxing of compound 2 with ethyl 2-(4-hydroxyphenylazo)-3-mercapto-3-phenylamino-acrylate $\mathbf{1 0}$ and sodium ethoxide in ethanol furnish the corresponding 3-hydroxy-2-(4-phenylthiazol-2-yl-carbamoyl)thiophene derivative 12. Presumably, the reaction started with nucleophilic attack of the thiol function resulting in the sulfide intermediate 11, and the following intramolecular cyclocondensation upon ethanol elimination afforded the corresponding 3-hydroxythiophene derivative $\mathbf{1 2}$ (Scheme 3).

4-( $p$-Hydroxyphenylazo)-3-methyl-5-phenylamino2-(4-phenylthiazol-2-yl-carbamoyl)thiophene 15 was obtained as the sole product by heating compound $\mathbf{2}$ with 3-( $p$-hydroxyphenylazo)-4-mercapto-4-(phenylamino)but-3-en-2-one (13) [11] and sodium ethoxide. The process was initiated by the nucleophilic attack of the thiol group and formation of sulfide intermediate 14, the following intramolecular cyclocondensation upon dehydration furnished the corresponding 3-methylthiophene derivative 15, the structure of which was supported by the spectral data (Scheme 4).

Molecular docking studies. COVID-19 discovered as the corona SARS-CoV-2 virus has a $\sim 30000$ nt RNA genome. It was translated into mature non-structural 


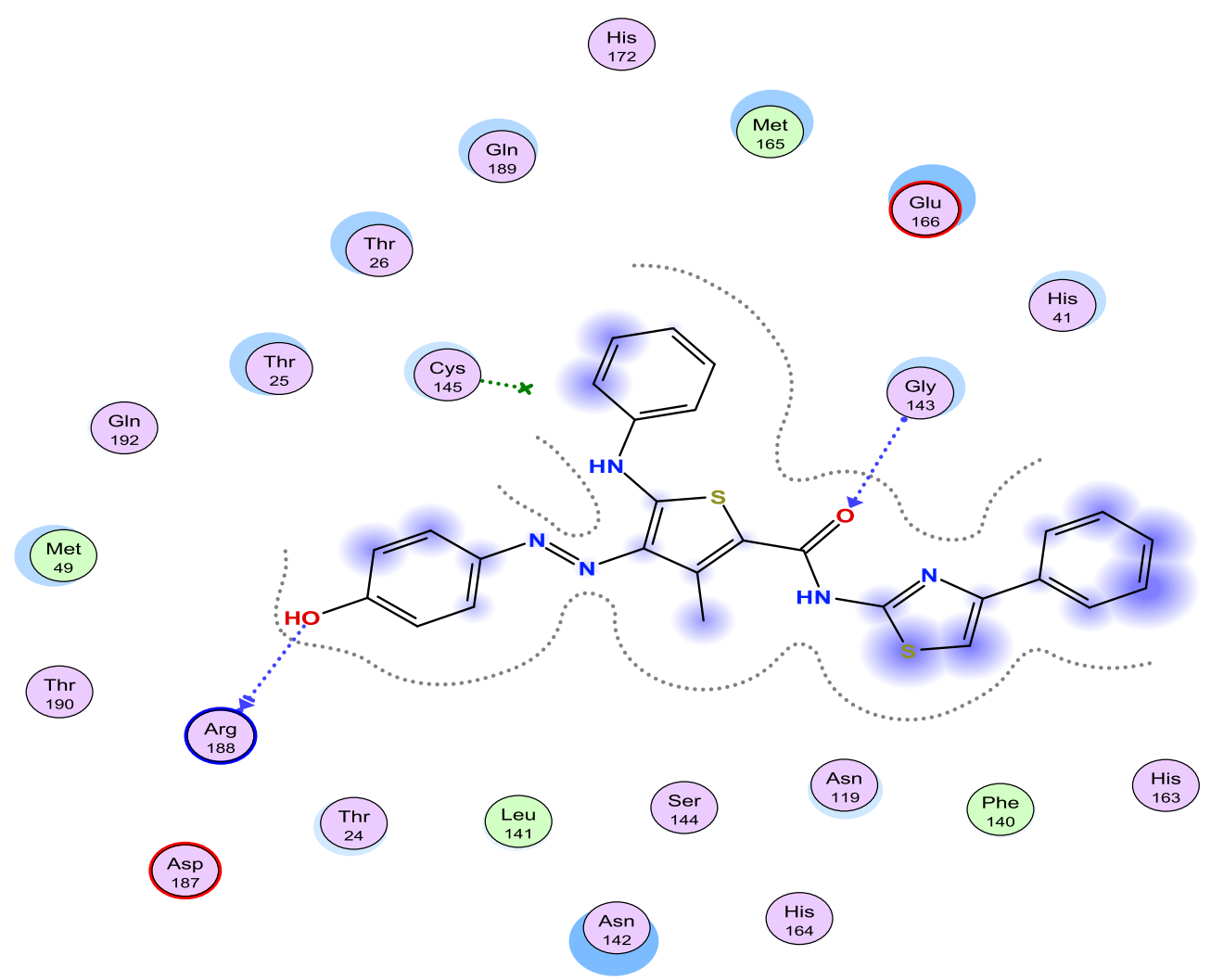

Fig. 1. 2D of the drug ligand interactions between compound $\mathbf{1 5}$ and $\mathrm{M}^{\text {pro }}$ enzyme active site.

proteins by an enzyme called the main protease $\left(\mathrm{M}^{\text {pro }}\right)$, which was proposed to be a good target in coronavirus therapy and discovery of new series of anti-coronavirus drugs [12-15].

Molecular docking was carried out by using the standard docking MOE2015.10 software. The "pdb file" of X-ray crystallographic structure of COVID-19 main protease ( $\mathrm{M}^{\text {pro }}$ ) (PDB ID: 7BUY) was obtained from the protein data bank (PDB). Our research group is experienced in the study of the drug-receptor interactions of a wide variety of newly synthesized molecules using molecular docking databases [16-22]. The presented docking protocol allowed us to calculate the important factor for interpretation of ligand-enzyme interactions between the synthesized heterocycles and $\mathrm{M}^{\text {pro }}$ enzyme in terms of E-score $(\mathrm{kcal} / \mathrm{mol})$ as follows: 1 (-4.91), 2 (-5.61), 3 (-5.93), 4a (-6.17), 4b (-5.98), 4c (-6.43), 5 $(-5.88), 6(-7.06), 9 \mathrm{a}(-7.23), 9 \mathrm{~b}(-7.04), 9 \mathrm{c}(-7.86), 12$ $(-7.80)$ and $15(-8.05)$. The data were compared with hydroxychloroquine as a reference molecule $(\mathrm{E}$-score $=$ $-6.50 \mathrm{kcal} / \mathrm{mol}$ ). The calculated data indicated that the protease active site protein residues were Asn 142, Thr
45, Thr 26, His 41, Glu 166, Thr 25, Gln 192, Thr 190, Ala 191, Arg 188, Leu 167, Met 165, Asp 187, Leu 141, Pro 168, Thr 42, Gly 143, His 164, Ser 144, Cys 145, Met 49, Gln 189, Ser 46, Cys 44, and Leu 27 (Fig. 1).

Most of the synthesized compounds were characterized by good interactions that formed two or more electrostatic bonds. The molecule $\mathbf{1 5}$ is a sample of the ligandenzyme electrostatic bonding (Fig. 2). The presented example demonstrates two hydrogen bonds between the carbonyl and hydroxyl groups of the molecule on one hand and Gly143 and Arg194 in the enzyme pocket on the other with the lengths measured as 2.23 and $1.94 \AA$, respectively.

\section{EXPERIMENTAL}

Melting points (uncorrected) were measured on a Gallenkamp electric melting point instrument. IR spectra (KBr disks) were recorded on a Thermo Scientific Nicolet iS 10 FT-IR spectrophotometer. ${ }^{1} \mathrm{H}$ and ${ }^{13} \mathrm{C}$ NMR spectra were measured on a Bruker WP $300 \mathrm{MHz}$ (Faculty of Science, Cairo University) using DMSO- $d_{6}$ as a solvent. 

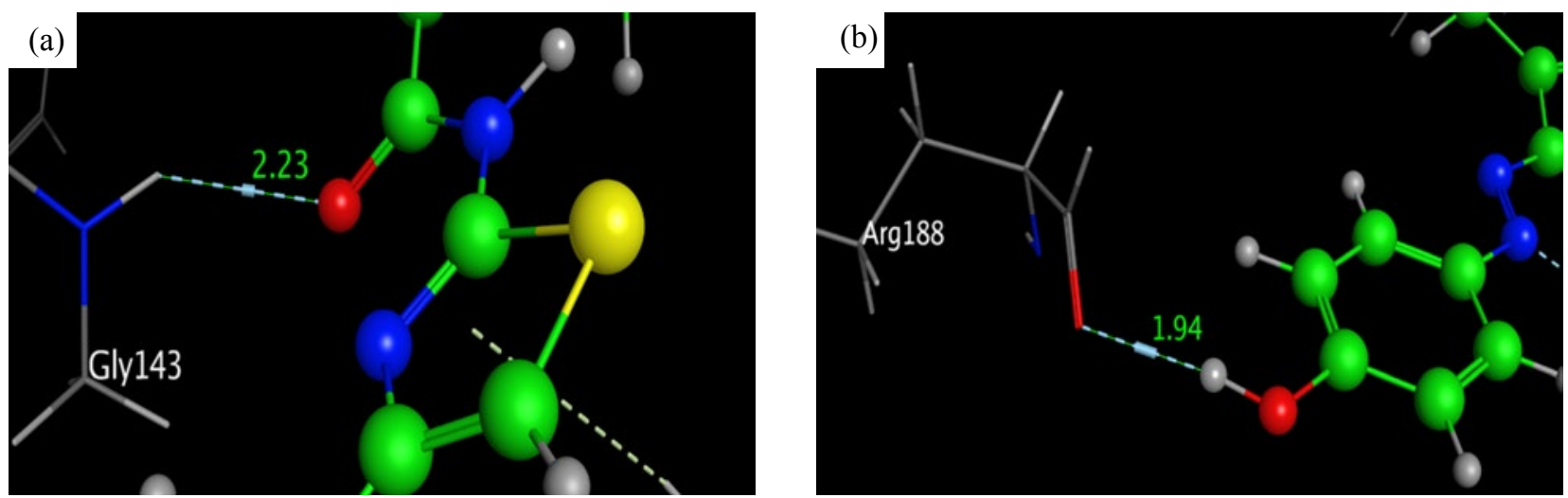

Fig. 2. The distances measured in $3 \mathrm{D}$ interaction diagrams of compound $\mathbf{1 5}$ with $\mathrm{M}^{\text {pro }}$ enzyme binding active sites: (a) H-bond with Gly143 and (b) H-bond with Arg188.

Mass spectra were measured on a Shimadzu Qp-2010 mass spectrometer (Faculty of Science, Mansoura University) at $70 \mathrm{eV}$ (EI mode). Elemental analysis was carried out on a Perkin-Elmer 2400 analyzer.

$\mathrm{N}$-(4-Phenylthiazol-2-yl)benzofuran-2-carboxamide (3). Salicylaldehyde ( $2 \mathrm{mmol}, 0.25 \mathrm{~mL}$ ) was added to a stirred solution of 2-chloroacetamido-4phenylthiazole (2) (2 mmol, $0.50 \mathrm{~g})$ in DMSO (10 mL) solution containing anhydrous $\mathrm{K}_{2} \mathrm{CO}_{3}(0.50 \mathrm{~g})$. The reaction mixture was stirred for $8 \mathrm{~h}$ at room temperature, then $10 \% \mathrm{HCl}$ solution $(20 \mathrm{~mL})$ was added, and the product was filtered off. The separated precipitate was recrystallized from ethanol. Yield $80 \%, \mathrm{mp} 234-236^{\circ} \mathrm{C}$. IR spectrum, $v, \mathrm{~cm}^{-1}: 3115(\mathrm{~N}-\mathrm{H}), 1676(\mathrm{C}=\mathrm{O}) .{ }^{1} \mathrm{H}$ NMR spectrum, $\delta$, ppm: $7.15-7.90 \mathrm{~m}\left(11 \mathrm{H}, \mathrm{H}_{\mathrm{Ar}}, \mathrm{C}^{5} \mathrm{H}_{\text {thiazole }}\right.$ and $\left.\mathrm{C}^{3} \mathrm{H}_{\text {furan }}\right), 11.15 \mathrm{~s}(1 \mathrm{H}, \mathrm{NH}) .{ }^{13} \mathrm{C}$ NMR spectrum, $\delta$, ppm: 106.25, 109.09, 112.38, 121.61, 123.26, 124.90, $126.85,127.60,128.35,129.58,133.43,148.20,151.44$, $156.84,162.67,163.72$. Found, \%: C 67.60; H 3.82; N 8.66. $\mathrm{C}_{18} \mathrm{H}_{12} \mathrm{~N}_{2} \mathrm{O}_{2}$ S. Calculated, \%: C 67.48; H 3.78; N 8.74. MS: $m / z: 320[M]^{+}$.

Synthesis of 2-amino-5-oxo-1-(4-phenylthiazol-2yl)-4,5-dihydro-3-substituted-1 $H$-pyrroles $(4 a-4 c)$. The appropriate substituted acetonitrile $(2 \mathrm{mmol})$ was mixed with sodium ethoxide $(0.09 \mathrm{~g})$ and compound 2 $(0.50 \mathrm{~g} ; 2 \mathrm{mmol})$ in ethanol $(20 \mathrm{~mL})$, and the mixture was refluxed for $6 \mathrm{~h}$. The mixture was poured into ice-cold water and then neutralized by dilute $\mathrm{HCl}$. The precipitate was filtered off and recrystallized from ethanol to give the corresponding pure product.

2-Amino-5-oxo-1-(4-phenylthiazol-2-yl)-4,5dihydro-1H-pyrrole-3-carbonitrile (4a). Yield 74\%, mp
274-275 ${ }^{\circ} \mathrm{C}\left(\mathrm{mp} 276-277^{\circ} \mathrm{C}\right.$ [23]). IR spectrum, $v, \mathrm{~cm}^{-1}$ : 3343, $3205\left(\mathrm{NH}_{2}\right), 2194(\mathrm{C} \equiv \mathrm{N}), 1737(\mathrm{C}=\mathrm{O})$.

2-Amino-5-oxo-1-(4-phenylthiazol-2-yl)-4,5dihydro-1H-pyrrole-3-carboxamide (4b). Yield 70\%, $\mathrm{mp} 184-186^{\circ} \mathrm{C}$. IR spectrum, $v, \mathrm{~cm}^{-1}: 3354,3194$ (broad, $\left.\mathrm{NH}_{2}\right), 1717(\mathrm{C}=\mathrm{O}$, pyrrole $), 1640(\mathrm{C}=\mathrm{O}$, amide $) .{ }^{1} \mathrm{H}$ NMR spectrum, $\delta$, ppm: $3.85 \mathrm{~s}\left(2 \mathrm{H}, \mathrm{CH}_{2}\right), 6.80 \mathrm{~s}(2 \mathrm{H}$, $\left.\mathrm{NH}_{2}\right), 7.40-7.85 \mathrm{~m}\left(6 \mathrm{H}, \mathrm{H}_{\mathrm{Ar}}\right.$, and $\left.\mathrm{C}^{5} \mathrm{H}_{\text {thiazole }}\right), 8.35 \mathrm{~s}(2 \mathrm{H}$, $\left.\mathrm{CONH}_{2}\right) .{ }^{13} \mathrm{C}$ NMR spectrum, $\delta$, ppm: 38.91, 87.24, 107.33, 127.15, 128.02, 129.48, 133.70, 148.62, 150.06, 161.25, 169.80, 173.55. Found, \%: C 56.16; H 3.98; N 18.74. $\mathrm{C}_{14} \mathrm{H}_{12} \mathrm{~N}_{4} \mathrm{O}_{2} \mathrm{~S}$. Calculated, \%: C 55.99; H 4.03; N 18.66. MS: $m / z$ : $300[M]^{+}$.

2-Amino-3-(benzothiazol-2-yl)-5-oxo-1-(4phenylthiazol-2-yl)-4,5-dihydro-1 $H$-pyrrole $(4 \mathrm{c})$. Yield $82 \%$, mp $148-150^{\circ} \mathrm{C}$. IR spectrum, $v, \mathrm{~cm}^{-1}: 3434$, $3315\left(\mathrm{NH}_{2}\right), 1754(\mathrm{C}=\mathrm{O}) .{ }^{1} \mathrm{H}$ NMR spectrum, $\delta$, ppm: $3.80 \mathrm{~s}\left(2 \mathrm{H}, \mathrm{CH}_{2}\right), 6.85 \mathrm{~s}\left(2 \mathrm{H}, \mathrm{NH}_{2}\right), 7.25-8.05 \mathrm{~m}(10 \mathrm{H}$, $\mathrm{H}_{\mathrm{Ar}}$, and $\left.\mathrm{C}^{5} \mathrm{H}_{\text {thiazole }}\right) .{ }^{13} \mathrm{C}$ NMR spectrum, $\delta$, ppm: 39.65 , $92.54,108.29,121.94,124.11,125.05,126.38,127.17$, 128.11, 129.43, 133.50, 136.23, 137.19, 147.88, 153.00, 156.73, 163.80, 174.48. Found, \%: C 61.41; H 3.57; N 14.26. $\mathrm{C}_{20} \mathrm{H}_{14} \mathrm{~N}_{4} \mathrm{OS}_{2}$. Calculated, \%: C 61.52; H 3.61; N 14.35. MS: $m / z: 390[M]^{+}$.

\section{2-[(4-Phenylthiazol-2-yl)imino]thiazolidin-4-one} (5). A mixture of solution of compound 2 ( $4 \mathrm{mmol}, 1.00 \mathrm{~g})$ in $20 \mathrm{~mL}$ of absolute ethanol and ammonium thiocyanate ( $6 \mathrm{mmol}, 0.46 \mathrm{~g}$ ) was refluxed for $5 \mathrm{~h}$ followed by cooling down to room temperature. The yellow precipitate was filtered off and dried. Yield $76 \%$, mp $257-259^{\circ} \mathrm{C}$. IR spectrum, $v, \mathrm{~cm}^{-1}: 3441(\mathrm{~N}-\mathrm{H}), 1727(\mathrm{C}=\mathrm{O}) .{ }^{1} \mathrm{H}$ NMR spectrum, $\delta$, ppm: $4.10 \mathrm{~s}\left(2 \mathrm{H}, \mathrm{CH}_{2}\right), 7.15 \mathrm{~s}(1 \mathrm{H}$, 
$\left.\mathrm{C}^{5} \mathrm{H}_{\text {thiazole }}\right), 7.35-7.80 \mathrm{~m}\left(5 \mathrm{H}, \mathrm{H}_{\mathrm{Ar}}\right), 12.15 \mathrm{~s}(1 \mathrm{H}, \mathrm{NH}) .{ }^{13} \mathrm{C}$ NMR spectrum, $\delta$, ppm: $34.98,112.08,127.33,128.19$, $129.40,133.72,149.83,159.11,172.69,175.53$. Found, \%: C 52.49; $\mathrm{H}$ 3.36; $\mathrm{N} 15.37 . \mathrm{C}_{12} \mathrm{H}_{9} \mathrm{~N}_{3} \mathrm{OS}_{2}$. Calculated, \%: C 52.35; H 3.29; N 15.26. MS: $m / z: 275[M]^{+}$.

5-(4-Chlorobenzylidene)-2-((4-phenylthiazol-2-yl)imino)thiazolidine-4-one (6). A mixture of the derivative 5 ( $2 \mathrm{mmol}, 0.55 \mathrm{~g})$ with $p$-chlorobenzaldehyde $(2 \mathrm{mmol}$, $0.28 \mathrm{~g})$ was refluxed in acetic acid $(25 \mathrm{~mL})$ containing anhydrous sodium acetate $(0.5 \mathrm{~g})$ for $4 \mathrm{~h}$, then cooled down and poured into ice-cold water. The solid precipitate was filtered off, dried and recrystallized form ethanol. Yield 58\%, mp $236-238^{\circ} \mathrm{C}$. IR spectrum, $v, \mathrm{~cm}^{-1}: 3134$ $(\mathrm{N}-\mathrm{H}), 1726(\mathrm{C}=\mathrm{O}) .{ }^{1} \mathrm{H}$ NMR spectrum, $\delta$, ppm: $7.10 \mathrm{~s}$ $\left(1 \mathrm{H}, \mathrm{C}^{5} \mathrm{H}_{\text {thiazole }}\right), 7.35-7.90 \mathrm{~m}\left(10 \mathrm{H}, \mathrm{H}_{\mathrm{Ar}}\right.$, and olefinic $\mathrm{C}=\mathrm{CH}), 11.65 \mathrm{~s}(1 \mathrm{H}, \mathrm{NH}) \cdot{ }^{13} \mathrm{C}$ NMR spectrum, $\delta$, ppm: 112.26, 117.36, 127.30, 128.16, 128.75, 129.20, 129.51, 132.93, 133.88, 134.02, 140.48, 150.13, 158.05, 170.18, 172.83. Found, \%: C 57.46; H 3.10; N 10.65. $\mathrm{C}_{19} \mathrm{H}_{12} \mathrm{ClN}_{3} \mathrm{OS}_{2}$. Calculated, \%: C 57.35; H 3.04; N 10.56. MS: $m / z: 397[M]^{+}$.

Synthesis of 5-phenylamino-2-(4-phenylthiazol-2yl-carbamoyl)-4-substituted-thiophene derivatives 9, 12, and 15. A mixture of compound 2 ( $2 \mathrm{mmol}, 0.50 \mathrm{~g})$ with the appropriate thiocarbamoyl derivative $7, \mathbf{1 0}$ or 13 ( $2 \mathrm{mmol})$ was refluxed for $4 \mathrm{~h}$ with sodium ethoxide solution $(0.09 \mathrm{~g})$ in $20 \mathrm{~mL}$ of absolute ethanol. The reaction mixture was cooled down to room temperature, poured into ice-cold water and neutralized by dilute $\mathrm{HCl}$. The precipitate was filtered off, dried and recrystallized from ethanol.

3-Amino-4-cyano-5-phenylamino-2-(4-phenylthiazol-2-yl-carbamoyl)thiophene (9a). Yield 64\%, mp $186-188^{\circ} \mathrm{C}$. IR spectrum, $v, \mathrm{~cm}^{-1}: 3313,3273,3192\left(\mathrm{NH}_{2}\right.$ and $\mathrm{NH}), 2201(\mathrm{C} \equiv \mathrm{N}), 1660(\mathrm{C}=\mathrm{O}) .{ }^{1} \mathrm{H}$ NMR spectrum, $\delta$, ppm: $6.75 \mathrm{~s}\left(2 \mathrm{H}, \mathrm{NH}_{2}\right), 7.15-7.85 \mathrm{~m}\left(11 \mathrm{H}, \mathrm{H}_{\mathrm{Ar}}\right.$, and $\left.\mathrm{C}^{5} \mathrm{H}_{\text {thiazole }}\right), 10.35 \mathrm{~s}(1 \mathrm{H}, \mathrm{NH}), 11.65 \mathrm{~s}(1 \mathrm{H}, \mathrm{NH}) .{ }^{13} \mathrm{C}$ NMR spectrum, $\delta$, ppm: 84.11, 107.63, 115.27, 119.02, $121.15,123.44,127.30,128.17,129.25,129.79,133.81$, 139.24, 147.92, 156.58, 157.33, 161.78, 163.47. Found, \%: C 60.56; H 3.66; N 16.70. $\mathrm{C}_{21} \mathrm{H}_{15} \mathrm{~N}_{5} \mathrm{OS}_{2}$. Calculated, \%: C 60.41; H 3.62; N 16.77. MS: m/z: $417[M]^{+}$.

3-Amino-4-carbamoyl-5-phenylamino-2-(4phenylthiazol-2-yl-carbamoyl)thiophene (9b). Yield $72 \%$, mp $218-220^{\circ} \mathrm{C}$. IR spectrum, $v, \mathrm{~cm}^{-1}: 3447,3410$, 3384, 3336, $3237\left(\mathrm{NH}_{2}\right.$ and $\left.\mathrm{N}-\mathrm{H}\right), 1635$ (broad, $\left.\mathrm{C}=\mathrm{O}\right) .{ }^{1} \mathrm{H}$ NMR spectrum, $\delta$, ppm: $6.35 \mathrm{~s}\left(2 \mathrm{H}, \mathrm{NH}_{2}\right), 7.10-7.85 \mathrm{~m}$ $\left(11 \mathrm{H}, \mathrm{H}_{\mathrm{Ar}}\right.$, and $\left.\mathrm{C}^{5} \mathrm{H}_{\text {thiazole }}\right), 8.75 \mathrm{~s}\left(2 \mathrm{H}, \mathrm{NH}_{2}\right), 10.85 \mathrm{~s}$
$(1 \mathrm{H}, \mathrm{NH}), 12.15 \mathrm{~s}(1 \mathrm{H}, \mathrm{NH}) .{ }^{13} \mathrm{C}$ NMR spectrum, $\delta, \mathrm{ppm}$ : $104.88,107.48,121.26,123.66,128.15,118.87,127.35$, 129.21, 129.70, 133.77, 139.21, 140.75, 148.05, 161.08, $162.91,163.58,166.39$. Found, \%: C 57.78; H 3.87; N 16.17. $\mathrm{C}_{21} \mathrm{H}_{17} \mathrm{~N}_{5} \mathrm{O}_{2} \mathrm{~S}_{2}$. Calculated, \%: C 57.91; H 3.93; N 16.08. MS: $m / z$ : $435[M]^{+}$.

3-Amino-4-(benzothiazol-2-yl)-5-phenylamino-2(4-phenylthiazol-2-yl-carbamoyl)thiophene (9c). Yield $82 \%$, mp $233-235^{\circ} \mathrm{C}$. IR spectrum, $v, \mathrm{~cm}^{-1}: 3429,3303$ $\left(\mathrm{NH}_{2}\right.$ and $\left.\mathrm{NH}\right), 1655(\mathrm{C}=\mathrm{O}) .{ }^{1} \mathrm{H}$ NMR spectrum, $\delta$, ppm: $6.70 \mathrm{~s}\left(2 \mathrm{H}, \mathrm{NH}_{2}\right), 7.10-8.10 \mathrm{~m}\left(15 \mathrm{H}, \mathrm{H}_{\mathrm{Ar}}\right.$ and $\left.\mathrm{C}^{5} \mathrm{H}_{\text {thiazole }}\right)$, $11.05 \mathrm{~s}(1 \mathrm{H}, \mathrm{NH}), 11.85 \mathrm{~s}(1 \mathrm{H}, \mathrm{NH}) .{ }^{13} \mathrm{C}$ NMR spectrum, $\delta$, ppm: 108.01, 120.17, 122.13, 122.48, 123.45, 124.06, $125.93,127.23,127.98,128.41,129.35,129.87,130.39$, 130.96, 133.81, 134.63, 139.22, 147.05, 147.92, 152.85, $154.25,162.21$, 163.70. Found, \%: C 61.84; H 3.71; N 13.43. $\mathrm{C}_{27} \mathrm{H}_{19} \mathrm{~N}_{5} \mathrm{OS}_{3}$. Calculated, \%: C 61.69; H 3.64; N 13.32. MS: $m / z: 525[M]^{+}$.

3-Hydroxy-4-(4-hydroxyphenylazo)-5-phenylamino-2-(4-phenylthiazol-2-yl-carbamoyl)thiophene (12). Yield $68 \%, \mathrm{mp}>300^{\circ} \mathrm{C}$. IR spectrum, $v, \mathrm{~cm}^{-1}$ : 3388 (broad $\mathrm{NH}$ and $\mathrm{OH}), 1613$ (broad, $\mathrm{C}=\mathrm{O}) .{ }^{1} \mathrm{H}$ NMR spectrum, $\delta$, ppm: $6.80-7.90 \mathrm{~m}\left(15 \mathrm{H}, \mathrm{H}_{\mathrm{Ar}}\right.$ and $\left.\mathrm{C}^{5} \mathrm{H}_{\text {thiazole }}\right)$, $9.90 \mathrm{~s}(1 \mathrm{H}, \mathrm{OH}), 11.85 \mathrm{~s}(1 \mathrm{H}, \mathrm{NH}), 12.75 \mathrm{~s}(1 \mathrm{H}, \mathrm{NH})$. ${ }^{13} \mathrm{C}$ NMR spectrum, $\delta$, ppm: 107.58, 116.27, 117.24, $120.28,123.69,126.93,127.28,128.51,129.33,129.78$, 131.06, 133.71, 134.13, 139.35, 147.92, 154.86, 156.39, 158.77, 163.20, 164.77. Found, \%: C 60.97; H 3.78; N 13.56. $\mathrm{C}_{26} \mathrm{H}_{19} \mathrm{~N}_{5} \mathrm{O}_{3} \mathrm{~S}_{2}$. Calculated, \%: C 60.80; H 3.73; N 13.64. MS: $m / z: 513[M]^{+}$.

4-(4-Hydroxyphenylazo)-3-methyl-5-phenylamino2-(4-phenylthiazol-2-yl-carbamoyl)thiophene (15). Yield $76 \%, \mathrm{mp} 278-280^{\circ} \mathrm{C}$. IR spectrum, $v, \mathrm{~cm}^{-1}: 3398$ $(\mathrm{NH}), 3297(\mathrm{OH}), 1635(\mathrm{C}=\mathrm{O}) .{ }^{1} \mathrm{H}$ NMR spectrum, $\delta$, ppm: $2.70 \mathrm{~s}\left(3 \mathrm{H}, \mathrm{CH}_{3}\right), 6.70-7.95 \mathrm{~m}\left(15 \mathrm{H}, \mathrm{H}_{\mathrm{Ar}}\right.$, and $\left.\mathrm{C}^{5} \mathrm{H}_{\text {thiazole }}\right), 9.90 \mathrm{~s}(1 \mathrm{H}, \mathrm{OH}), 12.25 \mathrm{~s}(1 \mathrm{H}, \mathrm{NH}), 13.18 \mathrm{~s}$ $(1 \mathrm{H}, \mathrm{NH}) .{ }^{13} \mathrm{C}$ NMR spectrum, $\delta$, ppm: 13.86, 107.52, $116.35,120.23,123.73,126.87,127.15,128.56,129.38$, $129.75,133.65,134.05,139.29,140.37,141.96,143.11$, $147.88,155.41,158.71,163.17,163.90$. Found, \%: C 63.25; H 4.21; N 13.80. Calculated, \%: $\mathrm{C}_{27} \mathrm{H}_{21} \mathrm{~N}_{5} \mathrm{O}_{2} \mathrm{~S}_{2}$. C 63.39; H 4.14; N 13.69. MS: $m / z: 511[M]^{+}$.

\section{CONCLUSIONS}

In summary, new thiazole-based derivatives have been synthesized by the reactions of 2-chloroacetamido4-phenylthiazole with various oxygen, carbon and/or sulfur nucleophilic reagents. Structures of the synthesized 
thiazole linked benzofuran, pyrrole, thiazolidine, and/or thiophene ring systems have been determined using IR, ${ }^{1} \mathrm{H}$ and ${ }^{13} \mathrm{C}$ NMR, and mass spectra. Docking procedure has exposed the more efficient interactions of the products 4c, 6, 9a-9c, 12, and 15 with COVID-19 main protease $\left(\mathrm{M}^{\mathrm{pro}}\right)$ binding site than hydroxychloroquine used as a reference ligand.

\section{FUNDING}

Taif University Researchers Supporting Project number (TURSP-2020/43), Taif University, Taif, Saudi Arabia.

\section{CONFLICT OF INTEREST}

No conflict of interest was declared by the authors.

\section{REFERENCES}

1. Zhu, X., Yang, Y., Xiao, G., Song, J., Liang, Y., and Deng, G., Chem. Commun., 2017, vol. 53, p. 11917. https://doi.org/10.1039/c7cc07366f

2. Abdel-Latif, E., Almatari, A.S., and Abd-ElGhani, G.E., J. Heterocycl. Chem., 2019, vol. 56, p. 1978. https://doi.org/10.1002/jhet.3577

3. Jacob, P.J. and Manju, S.L., Bioorg. Chem., 2020, vol. 100, p. 103882. https://doi.org/10.1016/j.bioorg.2020.103882

4. Sharma, P.C., Bansal, K.K., Sharma, A., Sharma, D., and Deep, A., Eur. J. Med. Chem., 2020, vol. 188, p. 112016. https://doi.org/10.1016/j.ejmech.2019.112016

5. Rettore, J.V.P., Oliveira, R.B.D., and Labanca, R.A., Braz. J. Pharm. Sci. 2019, vol. 55, p. e18218. https://doi.org/10.1590/s2175-97902019000118218

6. Kurt, B.Z., Gazioglu, I., Sonmez, F., and Kucukislamoglu, M., Bioorg. Chem., 2015, vol. 59, p. 80. https://doi.org/10.1016/j.bioorg.2015.02.002

7. Laine, L., Kivitz, A.J., Bello, A.E., Grahn, A.Y., Schiff, M.H., and Taha, A.S., Am. J. Gastro., 2012, vol. 07, p. 379. https://doi.org/10.1038/ajg.2011.443

8. Guay, D.R., Clin. Ther., 2002, vol. 24, p. 473. https://doi.org/10.1016/S0149-2918(02)85125-6

9. https://www.bbc.com/future/article/20200325-covid19-the-history-of-pandemics.

10. Geronikaki, A. and Theophilidis, G., Eur. J. Med. Chem., 1992, vol. 27, p. 709. https://doi.org/10.1016/0223-5234(92)90091-E
11. Habib, O.M., Moawad, E.B., Abdel-Latif, E., El-Mekabaty, A., and Sasi, D.S., Res. J. Pharm. Biolog. Chem. Sci., 2016, vol. 7, p. 128.

12. Hegyi, A. and Ziebuhr, J., J. Gen. Virol., 2002, vol. 83, p. 595.

https://doi.org/10.1099/0022-1317-83-3-595

13. Anand, K., Palm, G.J., Mesters, J.R., Siddell, S.G., Ziebuhr, J., and Hilgenfeld, R., EMBO J., 2002, vol. 21, p. 3213 .

https://doi.org/10.1093/emboj/cdf327

14. Yang, H., Yang, M., Ding, Y., Liu, Y., Lou, Z., Zhou, Z., Sun, L., Mo, L., Ye, S., Pang, H., and Gao, G.F., Proc. Nat. Acad. Sci., 2003, vol. 100, p. 13190. https://doi.org/10.1073/pnas.1835675100

15. Pillaiyar, T., Manickam, M., Namasivayam, V., Hayashi, Y., and Jung, S.H., J. Med. Chem., 2016, vol. 59, p. 6595. https://doi.org/10.1021/acs.jmedchem.5b01461

16. Abdelghany, A.M., Soliman, H.A., and Khatab, T.K., J. Organomet. Chem., 2021, vol. 944, p. 121847. https://doi.org/10.1016/j.jorganchem.2021.121847.

17. Khatab, T.K., Abdelghany, A.M., and Soliman, H.A., App. Organomet. Chem., 2019, vol. 33, p. e4783. https://doi.org/10.1002/aoc.4783

18. Khatab, T.K., Abdelghany, A.M., Kandil, E.M., Elsefy, D.E., and El-Mekabaty, A., Biointerface Res. Appl. Chem., 2020, vol. 10, p. 5182. https://doi.org/10.33263/BRIAC102.182187

19. Khatab, T.K., Ez-el-din, M.K., Elsefy, D.E., and El-Mekabaty, A., Biointerface Res. Appl. Chem., 2021, vol, 11, p. 13779. https://doi.org/10.33263/BRIAC116.1377913789

20. Soliman, H.A. and Khatab, T.K., Silicon, 2018 , vol. 10, p. 229. https://doi.org/10.1007/s12633-016-9421-0

21. Khatab, T.K., Mubarak, A.Y., and Soliman, H.A., J. Heterocycl. Chem., 2017, vol. 54, p. 2463. https://doi.org/10.1002/jhet.2846

22. Soliman, H.A., Khatab, T.K., and Abdel-Megeid, F.M., Chinese Chem. Lett., 2016, vol. 27, p. 1515. https://doi.org/10.1016/j.cclet.2016.03.026

23. Bondock, S., El-Azab, H., Kandeel, E.E.M., and Metwally, M.A., Synth. Comm., 2013, vol. 43, p. 59. https://doi.org/10.1080/00397911.2011.591036 